\title{
Ratios among atmospheric trace gases together with winds imply exploitable information for bird navigation: a model elucidating experimental results
}

\author{
H. G. Wallraff \\ Max Planck Institute for Ornithology, 82319 Seewiesen/Starnberg, Germany \\ Correspondence to: H. G. Wallraff (wallraff@orn.mpg.de)
}

Received: 1 July 2013 - Published in Biogeosciences Discuss.: 23 July 2013

Revised: 19 September 2013 - Accepted: 26 September 2013 - Published: 4 November 2013

\begin{abstract}
A model of avian goal-oriented navigation is described that is based on two empirical findings building a bridge from ornithology to atmospheric chemistry. (1) To orient their courses homeward from distant unfamiliar areas, homing pigeons require long-term exposure to undisturbed winds at the home site and olfactory access to the environmental air at home and abroad. (2) Above Germany, ratios among some atmospheric trace gases vary along differently oriented spatial gradients as well as depending on wind direction. The model emulates finding (1) by utilising the analysed air samples on which finding (2) is based. Starting with an available set of 46 omnipresent compounds, virtual pigeons determine the profile of relative weights among them at each of 96 sites regularly distributed around a central home site within a radius of $200 \mathrm{~km}$ and compare this profile with corresponding profiles determined at home under varying wind conditions. Referring to particular similarities and dissimilarities depending on home-wind direction, they try to estimate, at each site, the compass direction they should fly in order to approach home. To make the model work, an iterative algorithm imitates evolution by modifying sensitivity to the individual compounds stepwise at random. In the course of thousands of trial-and-error steps it gradually improves homeward orientation by selecting smaller sets of most useful and optimally weighted substances from whose proportional configurations at home and abroad it finally derives navigational performances similar to those accomplished by real pigeons. It is concluded that the dynamic chemical atmosphere most likely contains sufficient spatial information for home-finding over hundreds of kilometres of unfamiliar terrain. The underlying chemo-atmospheric processes remain to be clarified.
\end{abstract}

\section{Introduction}

This article deals with a problem that unexpectedly joins two disciplines which have never been considered to be in any way connected: avian behavioural biology and chemistry of the free atmosphere. The origin of the connection goes back to a short publication by Papi et al. (1971) who found that carrier pigeons with sectioned olfactory nerves were highly impaired in their homing performances. Forty years later we have a sizeable collection of coherent experimental results showing that experience of natural winds at the home site and smelling of airborne trace gases is a necessary precondition for homeward navigation of pigeons and other birds from unfamiliar areas over distances of some $50-300 \mathrm{~km}$ and more (for review and discussion of debated problems see Papi, 1986, 1989, 1991; Wallraff, 1990, 1996, 2001, 2004, 2005a b, 2010; Gagliardo, 2013; see also Able, 1996; Gagliardo et al., 2013).

The key results are as follows.(1) Pigeons made anosmic by sectioning their olfactory nerves and released in unfamiliar areas $150-300 \mathrm{~km}$ distant from home, fly, in contrast to home-oriented control birds, in arbitrary directions, often far away ( $>100 \mathrm{~km}$ air-line distance), and almost none return to the loft. (2) When pigeons, after sitting for an hour or more in an airtight container ventilated with outside air at the release site, are released under nasal anaesthesia, they behave differently depending on the pre-release conditions: if they had been allowed to smell natural local air, they depart homeward oriented; if the air sucked through the container had passed a charcoal filter removing airborne trace gases, they depart disoriented. (3) In analogous experiments in which the birds had been allowed to smell local air for $3 \mathrm{~h}$ at a distant site and were then transported, in filtered air, to a release site in 
the opposite direction from home, they depart preferentially to that compass direction which would have led them home from the site at which they had access to natural air. (4) If, during their long-term stay at home, pigeons had been kept in an aviary shielded from winds and are then released far away, they fly as disoriented as anosmic birds. Deflection or reversion of winds in the aviary results in correspondingly deflected or reversed flight courses upon release at remote sites. For a brief listing of additional results see Wallraff (2005b).

Having these experimental findings and the resulting conclusion that airborne volatile compounds perceived by olfaction comprise essential signals making home-finding over hundreds of kilometres possible, we have not yet gained an understanding of this kind of navigation, but merely opened a new, now more specific problem. How might olfaction-based long-distance navigation operate? It is obvious that, in contrast to other kinds of olfaction-based orientation, it is not a simple approach towards an odour source (homing not only against headwinds, distances $>100 \mathrm{~km}$ ). The system must be more sophisticated.

It appears to be a commonly accepted precondition for goal-finding using position-dependent local cues in an unfamiliar area that it requires reference to at least two coordinates, in physical terms gradients of quantifiable parameters, which intersect at sufficiently large angles (bi-coordinate or multi-coordinate navigation; e.g. Wallraff, 1990, 2004, 2005a; Able, 2000; Boström et al., 2012), so that coordinate values at a distant position can be compared with memorised home-site coordinates. Owing to the instability of the atmosphere with its highly variable wind and weather conditions, however, it appears, at first glance, inconceivable that any trace gases dispersed in that atmosphere might meet this requirement (intuitive common sense assessment; Becker and van Raden, 1986; Waldvogel, 1987, 1989). Nevertheless, a search for long-range gradients not of absolute concentrations of single volatile compounds, but of proportional relations between some of them, was successful (Wallraff and Andreae, 2000; henceforth cited as W\&A). Using such empirically determined ratio gradients, appropriately programmed model pigeons were able to steer homeward within a radius of $200 \mathrm{~km}$ at a level of precision quite comparable to that of real pigeons (Wallraff, 2000).

The calculations leading to these results were based on the assumption that the birds know the specific directions of some ratio gradients, but deferred the question of how they had achieved their knowledge. The ratio-gradient hypothesis of olfactory navigation, however, includes the mode of achievement (Wallraff, 1989, 2000, 2001, 2004, 2005a, b, 2010; Gagliardo, 2013). It includes, as a second necessary parameter of the system, the wind, i.e. a particularly dynamic variable which initially would have been expected to cause disturbance rather than providing useful information. However, as mentioned above, an essential precondition of pigeon homing over unfamiliar terrain is not only olfactory access to environmental air, but also long-term exposure to natu- ral winds at the home site. Therefore, the complete hypothesis includes the assumption that the birds associate, at home, varying olfactory input compositions with concurrently varying directions of the wind. Displaced to an unfamiliar distant site, they are thought to compare a specific olfactory sensation perceived there with the diverse sensations experienced at home and then decide to steer that compass direction towards which the wind at home blew while the olfactory input shared specific peculiarities of the current input at the best. Thereby, the birds might deduce the alignments of spatial gradients from olfactory experiences with varying winds at home. They need, however, not be aware of any gradients; it is sufficient to respond properly.

Atmospheric relationships observed by W\&A actually revealed a clear overall correlation of ratios among volatile organic compounds (VOCs) varying with wind direction, on the one hand, and of VOC ratios varying along spatial directions away from the centre of the investigated area (the "home site"), on the other hand. For most (not all) substances, however, the two directions were not identical; the wind axes deviated from the spatial axes by an average angle of about $45^{\circ}$ clockwise (Fig. 10 in W\&A). It remained unclear whether this fairly consistent angular divergence is generally representative or whether it might have resulted from a dominance of anticyclonic weather conditions or from other accidental circumstances during data acquisition. Therefore, a former model (Wallraff, 2000) left this aspect aside and operated with the spatial gradients only. The detection of their existence was the core outcome of W\&A which we considered, at that time, merely a first pilot study. I thought it advisable to wait with more advanced model calculations until more comprehensive data sets would be available. As, however, a more comprehensive data set is still lacking, I eventually tried to exploit the W\&A data in a more comprehensive way.

The present study aims to answer this question: to what extent can pigeons without knowledge of any conditions away from home, but experienced with winds and correlated specific olfactory sensations at home, potentially deduce information on the direction they should fly from trace gases perceived at various sites around their home loft at distances of up to $200 \mathrm{~km}$ ?

It was clear from the outset (and from W\&A) that only a fraction of the numerous VOCs dispersed in the atmosphere could in any way be useful as navigational signals. During evolution, the birds should have specialised themselves to use those compounds whose proportional quantity varies most regularly and correspondingly in space as well as with wind direction. They may have developed particular sensitivity to these substances and/or may give them particular weight during signal processing. We can expect that the ratio profiles actually evaluated by birds are most effectively adapted to optimal resolution of spatial and wind-correlated regularities in the chemical atmosphere. It is the aim of this study to search for such optimally operating evaluation systems by 
simplified imitation of evolutionary processes making use of the available atmospheric data. Although the analysed trace gases are merely substitutes for the unknown chemosignals hypothetically used by birds, they can well serve as a model basis extracted from the free dynamic atmosphere with all its spatio-temporal instabilities.

In the foreground, we have a biological problem: how do birds navigate? In the background, we have a problem of atmospheric chemistry, which appears not less intriguing: how is it possible that sufficiently stable and spatially far-reaching regularities in the proportional composition of a number of trace gases over the central European continent do exist? The model described below, using virtual birds as tools to analyse atmospheric data in an appropriate way, may help to approach this problem as well.

\section{Database and rationale of the model}

Air samples were collected at 96 sites symmetrically distributed within a radius of $200 \mathrm{~km}$ around a former pigeon loft near Würzburg (Germany) during 16 trips, each along two perpendicular axes at intervals of about $30 \mathrm{~km}$ with the centre included (see Fig. 1 in W\&A and below Fig. 7). In the course of three summers, each peripheral position was visited twice, the centre 32 times. The 224 air samples were analysed by gas chromatography as described by W\&A. As only more or less omnipresent VOCs can be useful for navigation, those 46 VOCs, out of more than 300 observed in total, were selected which had been recognised in at least $90 \%$ of the 224 chromatograms. In the following, these substances are simply denominated by consecutive numbers following the sequence of gas-chromatographic retention times and thus roughly according to increasing molecular weight. Of these 46 compounds, 21 have been chemically identified (Supplement 1; i.e. see first section of Supplement). During the $2 \mathrm{~h}$ of air sampling, winds were measured about $3.5 \mathrm{~m}$ above the ground. For more details see W\&A.

A ratio profile of a set of odorous compounds is characterised by the relative quantitative weights (intensities) of the stimuli induced by the included substances. These weights result from (1) the physical concentration in the atmosphere and (2) the biological efficacy. Animals are differently sensitive to different chemicals so that the stimulus elicited by substance A may be stronger than that of $\mathrm{B}$, although the physical concentration of $\mathrm{B}$ is much higher. The hypothesis proposes that during evolution the birds' sensitivity to the various atmospheric trace gases (or post-perceptive weighting) has been selectively adapted in such a way that a most effective combination of a number of optimally weighted compounds is implemented for navigation. The model imitates these evolutionary processes in a simplified way.

Like real homing experiments with pigeons, the model includes two actors. The virtual experimenter knows the geometrical pattern of the central home site surrounded by 96 pe- ripheral sites and its alignment within the compass rose, but he knows nothing about the atmosphere. He releases the second actor, a virtual pigeon (VP), at all or some of the peripheral sites and observes the compass bearings of its departures. He determines the angles between these bearings and the position-dependent true compass directions towards home. From the whole set of angular differences he computes a parameter indicating the overall level of homeward directedness. The VP, in contrast, knows nothing about the spatial distribution of release sites, but it remembers the olfactory sensations perceived at home with different compass directions of the wind (birds are known to have a sun compass (Kramer, 1952) and a magnetic compass (Merkel and Wiltschko, 1965); reviewed by, e.g. Wallraff, 2004, 2005a, 2010). It compares the wind-correlated ratio profiles observed at home with the olfactory input at the current release site. From adequate such comparisons it deduces the initial compass directions of its flight at many release sites, which the experimenter observes. The better the VPs had selected and evaluated the most suitable VOCs "over generations", the better are, at the end, their directional decisions.

\section{Procedural algorithms}

Prior to any specific calculations, the weight of each of the 46 VOCs was standardised to an average initial level of 1 , so that differences in overall levels of compound-specific physical concentration were balanced (Supplement 2). Chromatograms (CGMs) containing less than $90 \%=41$ of the 46 VOCs were discarded so that the sample of CGMs used for calculations is somewhat smaller than the sample collected (204 out of 224). Note that any reference to a CGM implies reference to a certain position on a certain day. All test positions away from the central position (home site) are called peripheral.

\subsection{The iteration loops: outline}

In order to exploit atmospheric signals most efficiently for navigational purposes, the VPs were programmed to improve their navigational performance by stepwise enhancing or reducing their sensitivity to the individual VOCs. As ratios among compounds are in the focus of interest, any change of relative stimulus intensity of one compound alters relative intensities of all other compounds as well. Therefore, it is impossible to optimise weighting scales individually VOC by VOC. It was necessary to run a long sequence of iteration loops (mostly $10^{4}$ ) during which the relative weight of each VOC was slightly altered up or down at random. Each new combination of differently weighted VOCs was then tested for its effectiveness as a source of positional information from which the VPs, when displaced to many symmetrically distributed peripheral sites, can deduce directions pointing, on average, more or less towards home. The 
criterion for effectiveness is the so-called overall homeward component $\overline{\bar{c}}_{\mathrm{H}}$ (range +1 to -1$)$ of the mean home-related vector resulting from many such directions as described below. Mean home-related vector and mean homeward component are equivalent to identically defined parameters as generally used in studies dealing with navigation of real pigeons (e.g. Wallraff, 2005a: Fig. 2.1 for a single release, Fig. 3.9 for means of a sample of mean directions; for details of related vector calculations see, e.g. Batschelet, 1981).

The first iteration loop always starts with $n=46$ VOCs, all having standardised average weight 1 . The initial fictitious homeward component $\overline{\bar{c}}_{\mathrm{H}}$ is set to its possible minimum -1 , so that afterwards it is always higher. Each loop consists of three phases.

Phase A. This phase simulates the long-term learning situation at home. Average VOC profiles (showing relative signal strength per VOC as a percentage of the sum of all included VOCs) linked with 12 overlapping wind sectors at home in angular steps of $30^{\circ}$ are determined. The VP stores these 12 wind-correlated profiles together with an overall mean home-site profile.

Phase $B$. This is the test phase in which a virtual experimenter releases a VP at each included peripheral site. The VP extracts a best possible estimate of a compass course towards home from one or another kind of comparison of the current local VOC profile with each of the 12 wind-correlated home profiles. The experimenter collects the resulting compass bearings, determines their angular deviations from the respective direct courses towards home and calculates a mean vector from all these deviations. The overall homeward component $\overline{\bar{c}}_{\mathrm{H}}$ of this vector is then the decisive outcome within a given iteration loop. Three modes of computing flight courses were applied of which Mode 2 was chosen as a standard method which always has been used if not otherwise stated.

Phase $C$. Here happen the above-mentioned small random alterations of average VOC weights from one VP generation to the next. If the homeward component resulting in phase B is higher than in any one of the preceding loops, the current test profile of weights is now taken as a new basis for the following series of random "mutations" producing a new average test profile. If $\overline{\bar{c}}_{\mathrm{H}}$ does not exceed the preceding levels, this VP generation dies off and the last pre-test VOC profile is again used as a basis of another series of random alterations. Thereafter the next loop starts with phase A (unless the preset number of iterations is reached). In the course of many repetitions of such loops, some VOCs gain more and more weight, while others lose weight and, when reaching zero, are discarded.

The whole cycle from phase $\mathrm{A}$ to phase $\mathrm{C}$ is henceforth referred to as an iteration step or ItStep. A defined sequence of many (mostly $10^{4}$ ) such ItSteps is called an iteration run or ItRun. A number of analogous ItRuns, each starting and operating under equal conditions with average VOC weights 1 , but each experiencing different sequences of random alterations in phase $\mathrm{C}$, is denominated an iteration series or ItSeries.

Examples of VOC profiles are shown in Supplement 3, Fig. S1. The phases A-C of each loop are described in more detail in the following sections.

\subsection{Loop phase A: determination of wind-related VOC ratios at the home site}

Like naive real pigeons, the VPs are thought to have been exposed to the atmosphere, before experimental displacement, only at their home site, i.e. at the centre of the investigated area. Unlike real pigeons, however, the VPs could not perpetually associate olfactory input patterns with concurrent directions of the wind, but could exploit only 21 CGMs obtained with wind velocities $\geq 4 \mathrm{~km} \mathrm{~h}^{-1}$. Wind directions were classified in 12 overlapping $\pm 90^{\circ}$ sectors at angular intervals of $30^{\circ}$. All winds arriving within a given semicircle had a component pointing towards the mid-sector direction whose value depended (a) on the angular deviation from that direction and (b) on wind velocity (for details see Supplement 4; preceding duration of that wind direction, which also might play some role, is neglected). At the end, each of the 12 classes of wind direction was associated with a respective average VOC profile (examples in Supplement 3, Fig. S1, "Wind at home").

In addition to the 12 wind-sector profiles, a no-wind profile was averaged from 9 air samples obtained with wind velocities $<4 \mathrm{~km} \mathrm{~h}^{-1}$. Further, from the $12+1$ single profiles an average home-site profile was calculated. Differences between wind-related values and home-site means were also determined.

For a particular algorithm described below (designated Mode 3), the 12 wind-sector directions $\omega_{\mathrm{s}}$ together with associated weights of a given VOC as scalar magnitudes $w_{\mathrm{s}, i}$ were treated as vectors. From these 12 vectors a second-order mean vector was computed giving the mean direction of wind $\bar{\omega}_{i}(i=1,2, \ldots, n ; n=$ number of VOCs $)$ under which the proportion of the particular VOC $i$ was highest. The length of the resulting vector, $\bar{r}_{i}$, indicates the degree of wind dependency (for vector calculations see Supplement 5). Small $\bar{r}_{i}$ means that the relative portion of the respective substance did not systematically, perhaps not at all, vary with wind direction, whereas large $\bar{r}_{i}$ indicates a strong connection with winds (see Supplement 3, Fig. S2).

\subsection{Loop phase B: determination of courses steered at surrounding distant sites}

When displaced to a given peripheral site, the VP compares in some way the VOC profile currently perceived there with 
corresponding observations it had made earlier, during phase A, under different wind directions at home. From these comparisons it deduces a best possible estimate of the compass bearing it should choose in order to reach its home site. From a sample of the deviations of such bearings from the respective true homeward direction at many sites, the overall homeward component $\overline{\bar{c}}_{\mathrm{H}}$ is computed giving the decisive measure of navigational performance. (Note that it makes no difference whether the bearings are thought to originate from the same individual or from a sample of many cloned VPs.)

The comparisons and the deductions can be conducted in different ways. Among a number of possible methods, three were applied, denoted Mode 1, 2 and 3. Unless something else is explicitly stated, results shown below were obtained with Mode 2.

\subsubsection{Mode 1: search for highest overall similarity of site-related and home-wind-related VOC profiles}

In a most simple approach, the overall difference between a certain VOC profile obtained at a certain peripheral position and each of the 12 wind-correlated home profiles is determined by accumulating the square differences between the individual VOC percentages. That wind-sector profile for which the overall difference is smallest is considered most similar to the current local profile and thus the VP is considered to be displaced to that compass direction (out of possible 12) from which the correlated wind arrived at home. Its reversal, towards which the wind blew at home, is the bearing $\alpha_{\mathrm{N}}$ towards which the VP starts flying. The experimenter observes this bearing and determines the angular difference to the direction towards home which is then stored as $\alpha_{\mathrm{H}}$. From $n$ values $\alpha_{\mathrm{H}}$ obtained at symmetrically arranged peripheral sites, a mean vector (Supplement 5 ) is calculated with direction $\bar{\alpha}_{\mathrm{H}}$, length $\bar{a}_{\mathrm{H}}$ and homeward component $\bar{c}_{\mathrm{H}}=\bar{a}_{\mathrm{H}} \cos \bar{\alpha}_{\mathrm{H}}$. This overall $\bar{c}_{\mathrm{H}}$ indicates the degree of homeward directedness of the initial bearings of the VP at a sample of sites around home. In analogy to the mostly used Mode 2, where the overall homeward component is a mean of means, the symbol $\overline{\bar{c}}_{\mathrm{H}}$ is henceforth used for $\bar{c}_{\mathrm{H}}$ obtained with Mode 1 as well.

Similar, slightly better results were achieved (but not shown) if not only the most similar home-wind profile was identified, but that axis of opposing winds that provides smallest differences in one direction and largest differences in the opposite direction.

\subsubsection{Mode 2: comparison of all home-wind-related and site-related differences from overall home-site means}

A profile of VOCs observed at a peripheral site can hardly have been observed in a really analogous form at home, as different VOCs may vary differently over distance as well as with winds along a given axis. Therefore, the more efficient
Mode 2 algorithm exploits the available data in a more comprehensive way. It does not compare VOC profiles directly, but their differences from an average home-site profile as determined in phase A. As key parameters it uses (a) these differences per VOC in the profiles obtained at the various peripheral positions and (b) coincidence or non-coincidence between the signs of these differences and of the corresponding differences observed at home with various wind directions. In the case of coincidence (both percentages above home-site mean or both below), the position-linked difference counts positive irrespective of its original sign, if the signs are opposed, it counts negative. Then, for a given home-wind direction, we add and average the newly signed differences of $n$ VOC weights at the respective position. The more the resulting mean deviates from zero, the higher is the probability that the VP has been displaced along the axis from which the wind at home blew while producing the respective homewind profile, either upwind or downwind. The mean difference is then taken as a scalar magnitude of a vector having the respective wind direction as angular component. Corresponding to the 12 home-wind classes, 12 such vectors are constructed for each included peripheral CGM.

In a second step, a site-specific mean compass vector is calculated whose direction depends on lengths and polarities (plus/minus) of the 12 primary vectors. It gives the direction toward which the VP assumes to be displaced. Its reversal by $180^{\circ}, \bar{\alpha}_{\mathrm{N}}$, gives the course it should fly. The angular deviation of this direction from the direction towards home, as determined by the experimenter, is $\bar{\alpha}_{\mathrm{H}}$. Vector length $\bar{a}_{\mathrm{H}}=\bar{a}_{\mathrm{N}}$ indicates the degree of certainty about the directional assessment (first vector calculation according to Supplement 5).

In a final step, a second-order mean vector $\overline{\bar{\alpha}}_{\mathrm{H}}$, length $\overline{\bar{a}}_{\mathrm{H}}$ is calculated from $n$ angles $\bar{\alpha}_{\mathrm{H}}$, where $n$ is the number of included CGMs obtained at peripheral sites (second vector calculation according to Supplement 5). With $\overline{\bar{c}}_{\mathrm{H}}=\overline{\bar{a}}_{\mathrm{H}} \cos \overline{\bar{\alpha}}_{\mathrm{H}}$ we achieve the crucial overall homeward component with its possible range from +1 to -1 .

The algorithm is described in more detail in Supplement 6. If not otherwise explicitly mentioned, the results shown below were obtained with this Mode 2 method which produced the clearest output.

\subsubsection{Mode 3: site-related differences from overall home-site means combined with wind directions of highest ratio increase at home}

Mode 3 is less powerful than Mode 2, i.e. it ends up with somewhat lower levels of homeward orientation. It is, nevertheless, included because it has the advantage of being transferable to other positions serving as home sites (Sect. 5.4). As a home-site reference, it uses only the average centralsite profile in which the 12 wind directions together with nowind are balanced (see phase A). In addition it uses the wind direction $\bar{\omega}_{i}$ derived in phase A (Sect. 3.2, last paragraph) as 
an estimated direction towards which the ratio of substance $i$ increases (see Supplement 3, Fig. S2).

Displaced to an unknown position $P$, the VP calculates the difference between relative VOC weights observed here and the corresponding mean values stored at the home site $H$ : $d_{\mathrm{P}, i}=w_{\mathrm{P}, i}-w_{\mathrm{H}, i}$. Depending on the sign of the difference, it creates a vector with length $\left|d_{\mathrm{P}, i}\right|$ and direction $\bar{\omega}_{i}$ (if $d_{\mathrm{P}, i}$ is negative) or $\bar{\omega}_{i}+180^{\circ}$ (if $d_{\mathrm{P}, i}$ is positive). Namely, if the current VOC weight $w_{\mathrm{P}, i}$ is lower than the remembered home weight $w_{\mathrm{H}, i}$, the VP should feel displaced downwind along the $\bar{\omega}_{i}$ axis, if it is higher, it should feel displaced upwind, i.e. towards the wind direction that was correlated, at home, with an increasing ratio of the particular compound. From $n$ (number of included VOCs) such single vectors, the VP calculates a mean vector whose direction $\bar{\alpha}_{\mathrm{P}, \mathrm{N}}$ gives the compass direction of the bird's flight (first vector calculation according to Supplement 5). The angle between $\bar{\alpha}_{\mathrm{P}, \mathrm{N}}$ and the direction towards home results as $\bar{\alpha}_{\mathrm{P}, \mathrm{H}}$. From the $\bar{\alpha}_{\mathrm{P}, \mathrm{H}}$ values obtained at all included positions, a final mean homeward vector results with direction $\overline{\bar{\alpha}}_{\mathrm{H}}$, length $\overline{\bar{a}}_{\mathrm{H}}$ (second vector calculation according to Supplement 5) and homeward component $\overline{\bar{c}}_{\mathrm{H}}$.

\subsection{Loop phase C: optimised weighting of chemosignals}

Independently of the method by which the overall homeward component $\overline{\bar{c}}_{\mathrm{H}}$ has been determined, the following algorithm of optimisation is always the same. Initially, when all 46 VOCs are included and considered with equal weight 1 , the overall homeward component $\overline{\bar{c}}_{\mathrm{H}}$ does not differ significantly from zero, i.e. the VPs are not recognizably homeward oriented. This is not surprising, as one cannot expect that every combination of so many equally weighted compounds is suitable for spatial guidance. How could birds have detected those VOCs that can serve as useful navigational signals while others should be ignored? Certainly they did so as evolution usually works: by trial and error over generations. And so works the model. It modifies the weights of the compounds at random as nature may have altered the birds' sensitivity to different substances by way of mutations. In thousands of iterative steps (ItSteps), the average weight $\bar{w}_{i}$ of each separate compound $i$ is more or less increased or decreased at random with small plus or minus changes being more frequent than somewhat larger ones (Supplement 7). In each such step of "mutations", a moderately altered profile of ratios among the compounds is generated. The efficiency of each new average profile is then tested by replication of phases A and B.

VOCs with increased weight affect the VPs' bearings of initial flight more than before, whereas the influence of VOCs with lower weight is reduced. If the overall homeward component $\overline{\bar{c}}_{\mathrm{H}}$ has decreased (as it happens most often), this profile is discarded and another series of random alterations of the hitherto used profile induced. If, however, $\overline{\bar{c}}_{\mathrm{H}}$ has increased, the new profile is accepted and serves as starting basis for the next step of alterations. During the re-

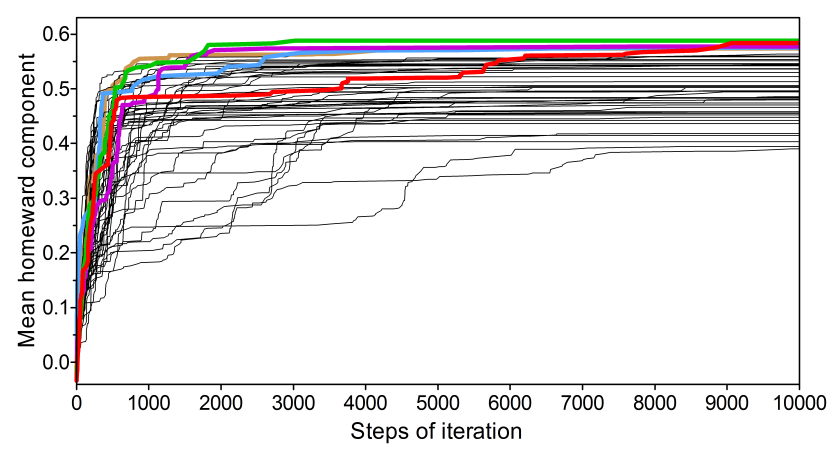

Fig. 1. Improvement of overall homeward components $\overline{\bar{c}}_{\mathrm{H}}$ in the course of 50 consecutive ItRuns (single lines), each consisting of $10^{4}$ ItSteps. The five ItRuns ending up with the highest values are highlighted by coloured lines. Included are 64 sites $90-200 \mathrm{~km}$ from the centre.

peated loops from phase A to C some of the VOCs gradually gain weight while others lose weight. The compounds with larger weights more and more dominate the calculations and the resulting vectors, while the others are more and more neglected. As soon as the weight of a particular VOC falls below zero, this VOC is removed from the sample of compounds. In this study, the iterative process routinely stopped after 10000 steps. The number of remaining compounds was then mostly reduced from 46 to $12-18$.

Depending on different sequences of random combinations, different ItRuns end up at different levels of the overall homeward component $\overline{\bar{c}}_{\mathrm{H}}$. For further considerations, only the top results are of interest, as they represent the best adapted selection and weighting of atmospheric chemosignals. Sequences of mutations leading to weaker performances would, in nature, not survive. Usually, we focus on the uppermost $10 \%$ of the achieved $\overline{\bar{c}}_{\mathrm{H}}$ values within an ItSeries.

\section{Basic results}

\subsection{Initial orientation in a range of $90-200 \mathrm{~km}$ around home}

The data obtained at 64 sites $90-200 \mathrm{~km}$ distant from the centre comprise 118 utilisable CGMs containing readable peaks of at least 41 of the 46 VOCs. At the beginning, with all VOCs having equal weight, $\overline{\bar{c}}_{\mathrm{H}}$ is close to zero, i.e. there is no trace of homeward orientation. Within the first thousand steps of random alterations, $\overline{\bar{c}}_{\mathrm{H}}$ usually increases quite rapidly, and after reaching a certain level, mostly little more progress occurs (Fig. 1). Finally, after $10^{4}$ ItSteps, most ItRuns end up with $\overline{\bar{c}}_{\mathrm{H}}$ between 0.4 and 0.6 ; the uppermost $10 \%$ are higher than 0.56 . Very few ItRuns, about $0.3 \%$, reach $\overline{\bar{c}}_{\mathrm{H}}>0.600 ; 20$ such top values have been achieved as a result of about 6500 ItRuns (Supplement 8). 


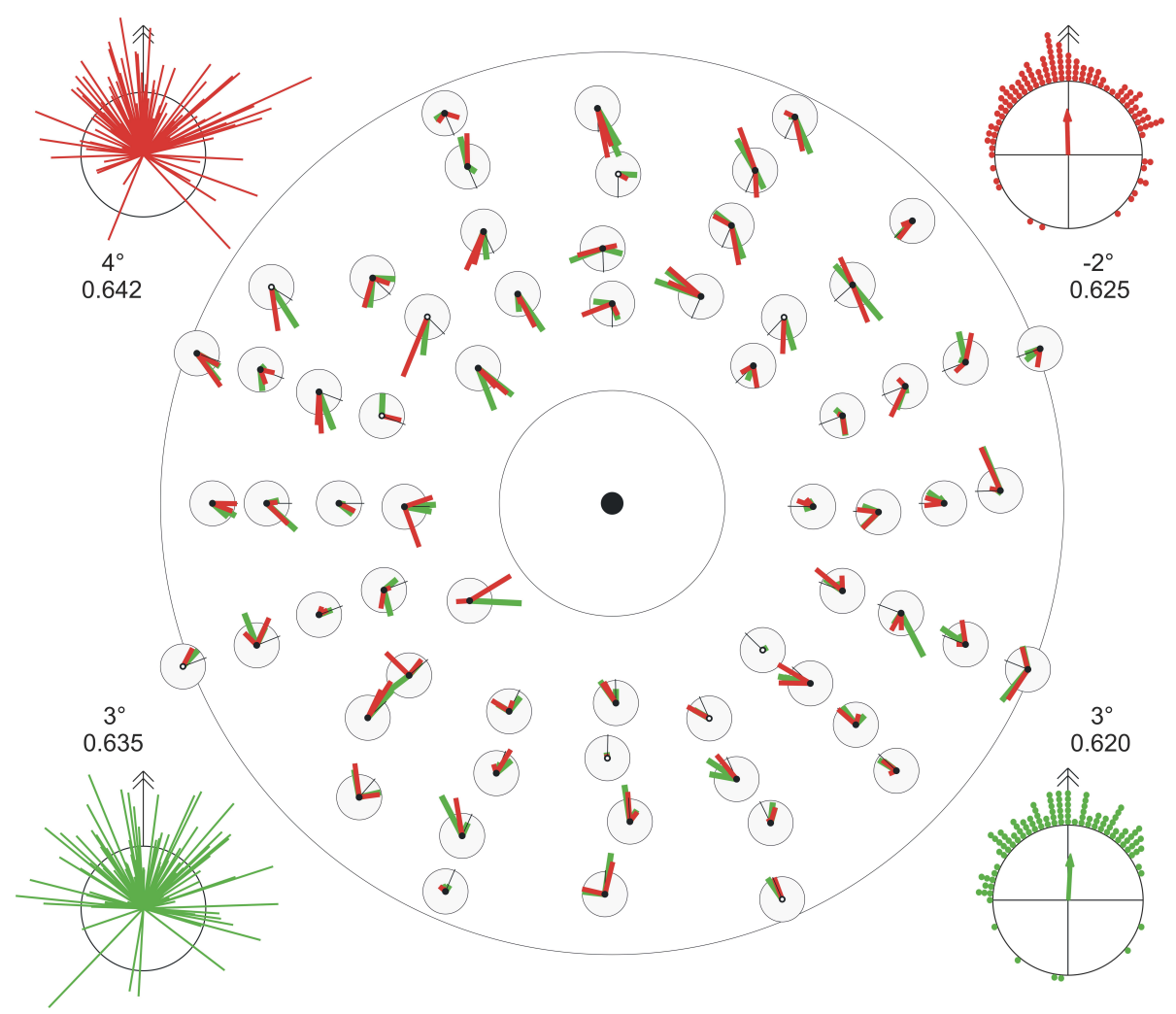

Fig. 2. Initial orientation vectors showing the results of the two most successful ItRuns (distinguished by colour) at 64 sites around the centre. Large diagram: radius of inner circle $50 \mathrm{~km}$, of outer circle $200 \mathrm{~km}$; north upwards. Radii of small circles at the different sites correspond to the median vector length of the whole sample (red or green, respectively; some vectors hidden by others). Two equally coloured vectors refer to the two chromatograms obtained in different years as available for most sites. If only one chromatogram could be used $(<90 \%$ of 46 VOCs determined), only one vector is shown (central dot white). Large circular diagrams: all $118 \overline{\bar{\alpha}}_{\mathrm{H}}$ values obtained at the 64 sites summarised, on the right the directions only (radius of circle $=1$ ), on the left the complete vectors (radius of circle $=$ median length $=1$ ); numbers (and central arrows in the right diagrams) give direction and length of resulting mean vectors. Direction towards home is upwards.

The individual vectors obtained with the two most successful ItRuns are shown in Fig. 2. They are comparable to vectors deduced from initial-flight bearings of real pigeons (see Supplement 9 and, e.g. Figs. 3.4 and 3.9 in Wallraff, 2005a). Varying lengths of vectors indicate varying clearness of directional decision, with real pigeons expressed as degree of angular scatter within a sample of independently departing individuals (vector length being a reciprocal measure of dispersion; short vector $=$ large angular scatter). Like real pigeons, the VPs are not perfectly homeward oriented, mostly they deviate more or less to the left or right, at some sites they are completely wrong or disoriented, but on the whole they show an explicit tendency to approach the centre. Results of the other top-20 ItRuns are similar (Supplement 9).

Many of the average weights of the finally remaining compounds are considerably above their original starting level around 1 (Fig. 3a-f). About two thirds of the 46 VOCs were eliminated in each of the 20 top ItRuns ending up with the highest navigational performance. The final profiles clearly differ from each other, no wonder, because each is the product of a different sequence of "mutations". Nevertheless, the profiles also show considerable similarities. In each profile the same 14 compounds were lacking, 15 of the remaining 32 had survived in more than half of the 20 ItRuns (green columns $>50 \%$ in Fig. 3f) and seven VOCs reached the final stage in all 20 ItRuns (100\% in Fig. 3f). The magnitudes of the remaining VOCs were more or less variable as well (red columns and black squares in Fig. 3f). These results indicate that variable combinations and variable weightings may lead to similar navigational success, but that, nevertheless, some trace gases are generally better suited as navigational signals than others and many are entirely unsuited. The clear favourite was VOC \# 7 (=2,2-dimethylbutane), which reached the highest weight in all most successful ItRuns (slope of ratio gradient increasing towards the SE; see Fig. S2 and see also VOC C5.3 in W\&A).

A good deal of navigational improvement can be achieved by pure selection of the most suited VOCs without optimised individual weighting. If those 16 or 14 compounds which finally remained in 50 or $60 \%$ of the top ItRuns were selected without giving them different weight, $\overline{\bar{c}}_{\mathrm{H}}$ was immediately as high as 0.38 , while its original starting level with all 


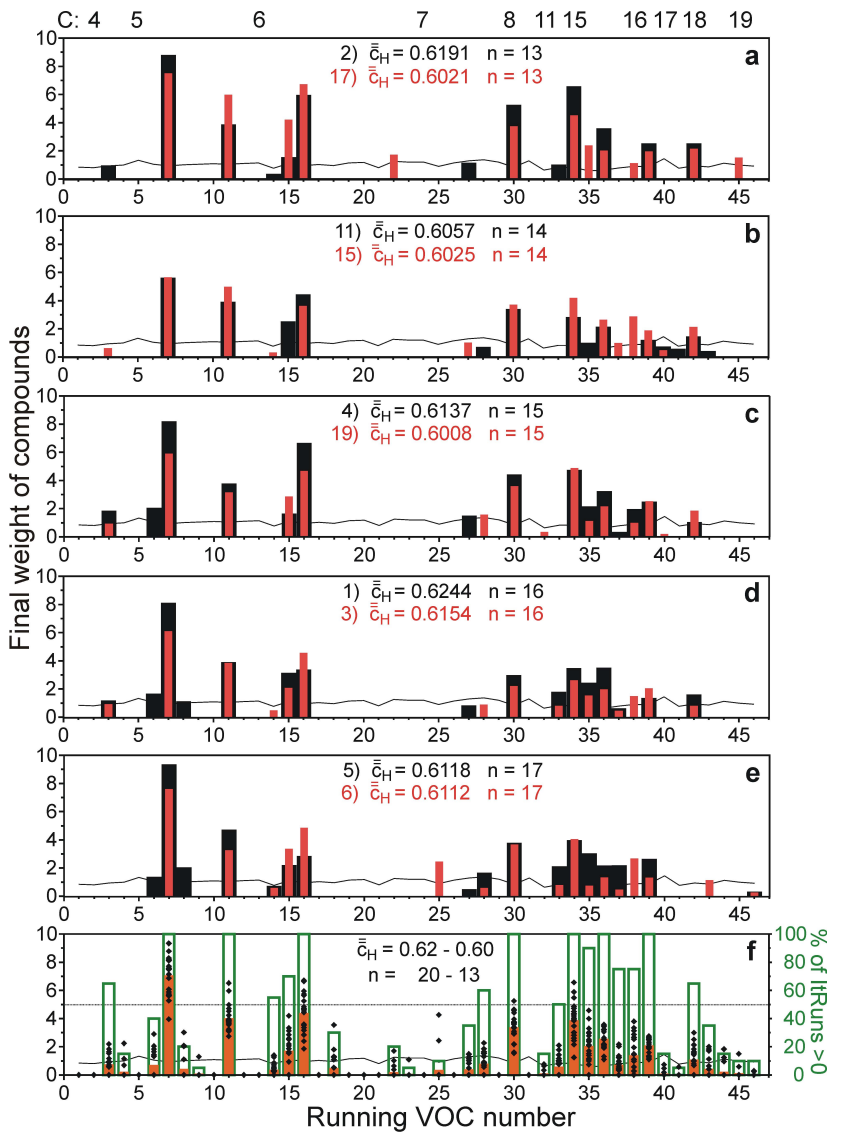

Fig. 3. (a-e) End stages of average weights of the 46 compounds (VOCs) in ten of the 20 ItRuns that reached the highest $\overline{\bar{c}}_{\mathrm{H}}$ values. Each diagram contains the results of two ItRuns (one drawn black and one red) with an equal number $(n)$ of remaining VOCs. Leading numbers indicate the rank position (1-20) of $\overline{\bar{c}}_{\mathrm{H}}$. The undulating thin line around 1 gives the initial standardised values from which the ItRuns started. (f) Black symbols give 1-20 weights per VOC\#, red columns resulting means (with zero values included). Green columns (right ordinate) give percentage of 20 ItRuns in which the respective VOC reached the end of $10^{4}$ ItSteps. Numbers on top give related positions of $\mathrm{n}$-alkanes with number of $\mathrm{C}$ atoms as indicated (see Table S1).

46 VOCs included was at -0.03 . ItRuns starting with such a reduced VOC sample from $\overline{\bar{c}}_{\mathrm{H}}=0.38$ can also reach final $\overline{\bar{c}}_{\mathrm{H}}$ values above 0.6 , ending up with $13-15$ remaining VOCs. However, with less than 13 VOCs, the reachable final level was $\overline{\bar{c}}_{\mathrm{H}}<0.54$. Thus, to reach optimal performance, the minimum number of exploited compounds was 13 (Supplement 10, Fig. S6).

\subsection{Directional specificity of wind experience at home}

It might appear conceivable that results like those shown above can similarly be achieved with any attribution of arbitrary or systematically false wind directions to the air samples collected at the central site. To some degree, this is actu-

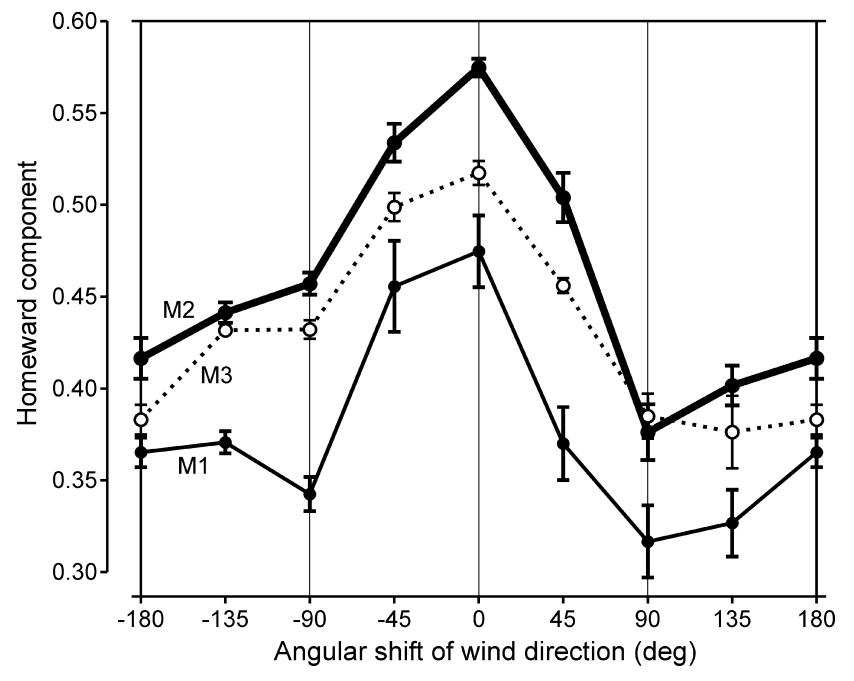

Fig. 4. Means ( $\pm 95 \%$ confidence limits) of uppermost 10 of 100 homeward components $\overline{\bar{c}}_{\mathrm{H}}$ obtained with correct linkage of VOC data and wind data at home $\left(0^{\circ}\right)$ and with wind directions shifted to the left (minus) or right (plus). M1, M2, M3 indicate phase B algorithm Mode 1, 2, 3, respectively. Data collected at distances 90-200 km (M2 at $0^{\circ}$ refers to an extra ItSeries of 100 ItRuns, not included in Supplement 8).

ally the case. For instance if, at home, the atmospheric data were associated not with the true concurrent wind, but with wind directions consistently shifted by a certain angle, top values of $\overline{\bar{c}}_{\mathrm{H}}$ still reached levels of more than 0.4 (Fig. 4, M2). However, they never reached a level as high as obtained with real winds correctly attributed (shift value $0^{\circ}$ ).

In order to guard against misunderstandings, it should be noted that these virtual wind shifts are not analogous to real wind deflections experimentally produced in so-called deflector aviaries (e.g. Papi, 1986; Wallraff, 2005a). The shifts constructed by the model took place during virtual development of signal weighting over generations. The VPs were instructed to develop optimal orientation towards true home using chemical profiles they had experienced, at home, while winds arrived from a direction rotated by a given angle to the left or right. (Or, inversely seen, the VPs were instructed to fly a certain angle to the wind direction they had associated with a given VOC profile.) With a shift angle of $-90^{\circ}$, for instance, the VPs correlate real west-wind VOC profiles with winds from the north, real north-wind profiles with winds from the east, etc., and try to optimise their orientation towards true home by modifying VOC weights as good as possible. If real wind conditions at home would not imply any information about spatial configurations, each shifted wind pattern would have the same chance of producing higher or lower levels of $\overline{\bar{c}}_{\mathrm{H}}$; a maximum level could be at any angle.

Figure 4 shows that not only the most efficient Mode 2 algorithm, but also Modes 1 and 3 operate best with the natural linkage between chromatographic results and concurrent 
direction of the wind during data sampling. The fact that the curves are not completely symmetric around zero might indicate some context with the $45^{\circ}$ divergence between spatial and wind-dependent variations of VOC ratios as mentioned in the Introduction. It has not been tested whether such a context exists.

\subsection{Statistical validation}

The findings shown in Fig. 4 demonstrate that the top results of homeward orientation cannot be achieved with any arbitrary coupling of central wind directions and VOC profiles. Nevertheless, the actually measured data might have provided particularly favourable combinations, producing the peak in Fig. 4 just at $0^{\circ}$, merely by chance. To test for this possibility, the real linkages between VOC profile and sampling position or between VOC profile and central wind direction have been replaced by variously combined linkages determined at random. If among many series of iterations analogous to those shown above, each based on different sets of randomly combined data, none or very few lead to homeward orientation on a level similar to that shown in Figs. 2 and 4 , it is obvious, or at least most likely, that those figures reflect real interrelations existing in nature.

Such tests are described in detail in Supplement 11. They provide clear evidence that spatially randomised positioning of VOC profiles can never lead to $\overline{\bar{c}}_{\mathrm{H}}$ values reaching the range achieved with the natural distribution. Thus, the existence of atmospheric ratio gradients, as revealed by W\&A, is a necessary precondition to let the model operate. Somewhat less certain is the dependence on natural winds. In 200 tests matching home-site winds with home-site CGMs at random, only four combinations $(=2 \%)$ led to $\overline{\bar{c}}_{\mathrm{H}}$ values that almost reached, reached or exceeded the range of $\overline{\bar{c}}_{\mathrm{H}}$ levels resulting with the real matches. It is not only logical, but also empirically confirmed (Supplement 11, Fig. S7, thin lines) that each of the eight investigated angular linkages between winds and chemical air data (Fig. 4) has the same probability of coming about by chance. Thus, the probability that just the actually observed linkage $\left(0^{\circ}\right.$ in Fig. 4) leads to maximum results by chance is an eighth of $2 \%$. Even if we would concede 5 instead of $2 \%$ (because 4 of 200 has its own range of possible error), the random probability of just the observed linkage is less than one percent.

These control tests confirm that not only the atmospheric data imply meaningful spatial and wind-correlated information, but also that the applied algorithm operates properly. Otherwise it would hardly have been possible to achieve successful simulation of pigeon homing at all.

\section{More specific results}

The ratio-gradient hypothesis implies three specific predictions. (1) With increasing distance from home, homeward orientation should become better, at least initially, because deviations from the average olfactory home profile should become greater and more distinct. (2) Homeward orientation should be better with tailwinds rather than with headwinds, because tailwinds should enhance and headwinds should diminish direction-specific proportional alterations. (3) Consequently, the wind effect should be greater at the shorter distances, because its proportional fraction to changes of ratio profiles should decrease with increasing distance.

In a final section we ask whether optimised VOC weightings achieved for homing to the central site are useful for homing to other sites as well.

\subsection{Effect of distance from home}

Sites of air sampling were distributed over distances of 30 $200 \mathrm{~km}$ from the centre near Würzburg. The iteration algorithms described above were applied to three distance classes, each containing 32 sites at which air samples for 64 CGMs were collected. Two equally sized overlapping classes were analysed in addition. Figure 5 shows a clear dependence of the achievable level of homeward orientation on distance. The level is quite low in the range $30-80 \mathrm{~km}$ (mean $55 \mathrm{~km}$ ) and possibly reaches a plateau somewhere beyond $100 \mathrm{~km}$. The dashed curve resulted from independent iterative optimisations for each of the five distance ranges (10 top $\overline{\bar{c}}_{\mathrm{H}}$ values of $100 \mathrm{ItSeries}$ consisting of $10^{4}$ ItRuns). The solid-line curve is based on the weighted VOC profiles of the ten top results gained from 100 ItSeries using the total of 192 (actually usable 174) CGMs obtained from the whole data sample including all sites from 30 to $200 \mathrm{~km}$. They were not specifically developed within the respective distance range, but applied only to the sites within this range.

These results fit the expectation that the model should work better over longer distances, because direction-specific differences should increasingly outweigh general noise the farther away from each other the two related positions are. The findings agree with the observation that distinct gradients of VOC ratios become apparent only farther away from the centre (Wallraff, 2005a, Fig. 7.36).

\subsection{Effect of current wind at the peripheral sites}

For analysis, the homeward component $c_{\mathrm{HW}}$ of current wind during air sampling was determined as $c_{\mathrm{HW}}=v \cos (\gamma-\beta)$, where $v$ is wind velocity in $\mathrm{km} \mathrm{h}^{-1}, \gamma$ the compass direction from which the wind comes, and $\beta$ the compass direction of the sampling site from the centre (or $\gamma$ the direction towards which the wind blows and $\beta$ the direction towards home). The 192 CGMs obtained from samples collected at all peripheral sites were subdivided into three classes according to current wind condition with respect to the homeward direction: tailwind - neutral wind - headwind. A threshold value of $c_{\mathrm{HW}}$ determined the borders between the classes. The actually used threshold of $3 \mathrm{~km} \mathrm{~h}^{-1}$ defines tailwind as 


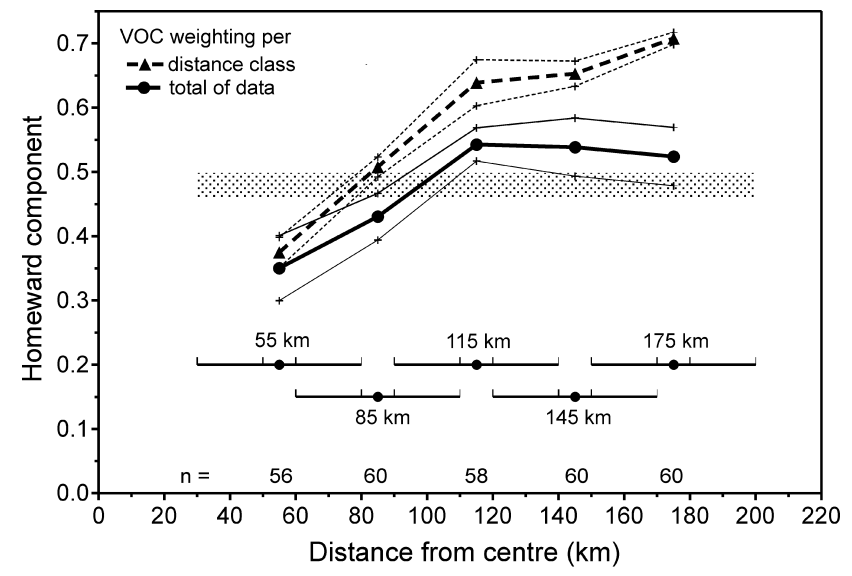

Fig. 5. Dependence of homeward orientation on distance from the centre. Means ( $\pm 99 \%$ confidence limits) of uppermost 10 of 100 homeward components $\overline{\bar{c}}_{\mathrm{H}}$ obtained with air data collected within varying distance ranges as indicated ( 8 sites per distance as marked by an upwards dash, 64 air samples per distance class). The brokenline results were achieved with separate optimisations per distance class starting with standardised VOC weights around 1 . The solidline calculations used VOC weightings achieved with the total data set ranging from 30 to $200 \mathrm{~km}$ and now applied to the respective distance class. Sample sizes $n$ of actually usable CGMs are given on the bottom. The dotted area indicates the $\overline{\bar{c}}_{\mathrm{H}}$ range of the uppermost $10 \%$ ItRuns summarising data of all 96 sites together.

$c_{\mathrm{HW}} \geq 3 \mathrm{~km} \mathrm{~h}^{-1}$, neutral wind as $-3<c_{\mathrm{HW}}<3$, and headwind as $c_{\mathrm{HW}} \leq-3 \mathrm{~km} \mathrm{~h}^{-1}$. Neutral wind can thus result from no or very weak wind or from crosswinds blowing neither from nor towards the centre.

In accordance with the hypothetical expectation, tailwinds appear favourable for home-finding and headwinds detrimental (Fig. 6, black symbols). Again, as in the case of distance, VOC weightings derived from the whole data sample lead, understandably, to less clear-cut results than weightings derived from the subsamples specifically selected with regard to wind conditions. Headwinds, at least, are correlated with weak performances achieved with either kind of calculation.

\subsection{Effects of distance and current wind combined}

Subdivision of the available data set according to both distance and wind leads to quite small sub-subsamples. If we subdivide distance in only two classes, we end up with samples including only 24-37 CGMs, so that accidental noise is less reliably balanced. In addition, the sites per sample are not as symmetrically distributed around the centre as the nonselected sites are within each distance class. Nevertheless, dependence on distance as well as on wind direction is still indicated (grey curves in Fig. 6). The results are compatible with two predictions: (a) homeward orientation is better with tailwind than with headwind at any distance. (b) At short distances, the wind effect is higher than at longer distances. $\Delta \overline{\bar{c}}_{\mathrm{H}}$ between tailwind and headwind is 0.22 at the shorter dis-

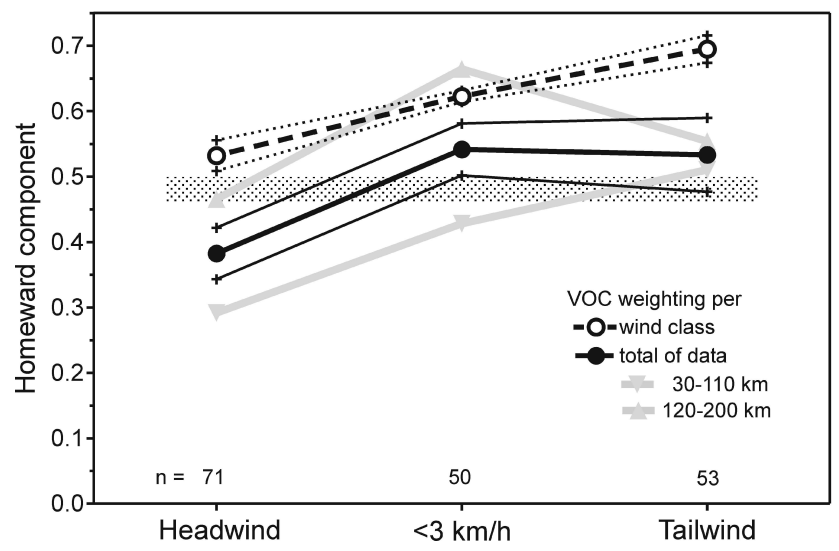

Fig. 6. Dependence of homeward orientation on direction of current wind. Black: means ( $\pm 99 \%$ confidence limits) of uppermost 10 of 100 homeward components $\overline{\bar{c}}_{\mathrm{H}}$ obtained with air data collected at all 96 sites with winds blowing more or less homewards or away from home. The broken-line results were achieved with separate optimisations per current wind condition starting with standardised VOC weights around 1 . The solid-line calculations used VOC weightings achieved with the total data set ranging from 30 to $200 \mathrm{~km}$ and now applied to the respective wind condition. Sample sizes $n$ of actually usable CGMs are given on the bottom. The dotted area indicates the $\overline{\bar{c}}_{\mathrm{H}}$ range of the uppermost $10 \%$ summarising data of all wind conditions together. Grey: solid-line results subdivided into two classes of distance from the centre.

tances and 0.09 at the longer distances. This outcome makes sense, because at short distances, with less distinct spatial differences of air conditions, the modifying effect of wind should be more pronounced. Owing to the restrictions mentioned above and owing to the less appropriate neutral-wind results, however, these results should be seen as supporting indication rather than as evidence.

\subsection{Homing to various sites}

As an evolutionary model, the above iterative algorithm is unrealistic insofar as it optimises homing to only one site, the centre of the investigated area. In real life, however, birds may establish their home territory at any place on earth and hence should be able to navigate to that optional place from everywhere within a large area around. Adaptively developed weighting of atmospheric signals should have led to more generally applicable profiles. The available data set arranged around one position with variable wind data is unsuited to mimic evolutionary processes based on cross-country movements in any order and covering much wider ranges.

Nevertheless, it can be tested whether optimised weighting developed for homing to one particular position improves homing to other sites as well. Established weighting scales of VOCs developed, as described above, for homing to the central site were used to ascertain whether their efficiency for homing to selected peripheral sites is correlated with their original level of efficiency for homing to the centre. In a 

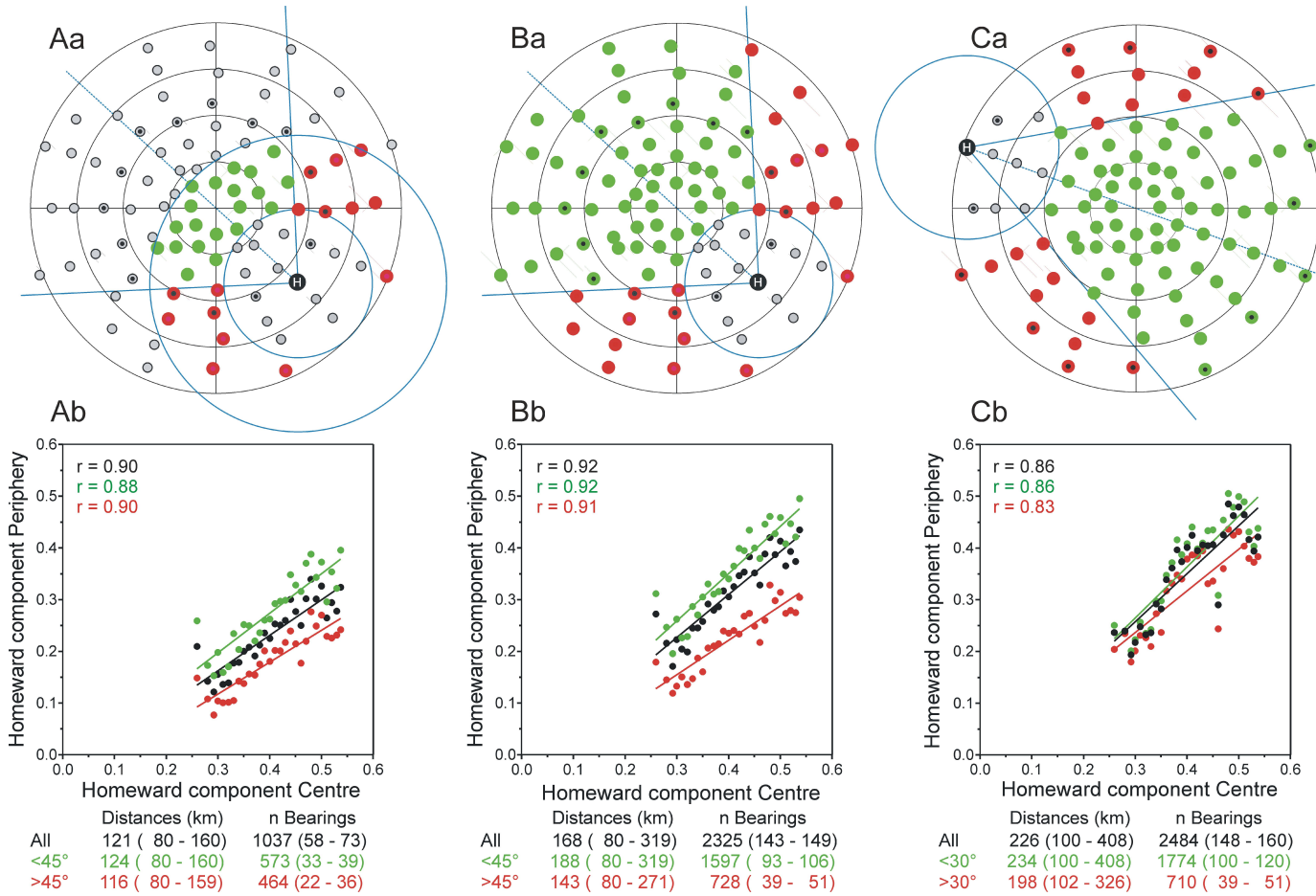

Fig. 7. Orientation towards peripheral goal sites. (Aa-Ca) (distance of circles from centre 50-200 km): three modes of selection of sampling sites. A black dot labelled " $\mathrm{H}$ " indicates the current home site in the case of a particular example. Blue circles and lines divide the surrounding sites into sections according to distance and angle from $\mathrm{H}$. Green are included sites within an angle of $\pm 45^{\circ}$ or $\pm 30^{\circ}$, red are included sites outside of this sector. Grey sites are not included in the calculations. In addition to the sites labelled $\mathrm{H}, 15$ more sites in each diagram at a similar distance, marked by a small black dot, were used as corresponding home sites with corresponding geometrical subdivisions around. (Ab-Cb) Correlations between 28 original centre-related $\overline{\bar{c}}_{\mathrm{H}}$ values at regular steps from 0.26 to 0.54 (abscissa) and mean homeward components related to 16 peripheral home sites $\mathrm{H}$, in each of the 28 cases (data points in the graph) using the same weighted VOC profile (ordinate). Below the graphs: means and ranges of distance between start and goal site and number of included VP bearings (in total and range per home site).

certain sequence, one of the peripheral sampling sites was defined as a current home site (see Fig. 7, Aa-Ca, black point "H"). Within a defined distance range, VOC patterns obtained at other sites (drawn green and red) were compared with the pattern at that home site in an analogous way as it has formerly been done, using Mode 3 (Sect. 3.3.3), with regard to the central site. Differences per compound were calculated on the basis of weighted profiles (cf. Fig. 3) derived from optimisations for homing to the centre. Such profiles and related final $\overline{\bar{c}}_{\mathrm{H}}$ values were available from 500 ItRuns (see Supplement 8, Fig. S4: M3). From these 500 ItRuns, 28 were selected at regular 0.01 intervals of $\overline{\bar{c}}_{\mathrm{H}}$ ranging from 0.54 to 0.26 . The related VOC profiles were then used to calculate homeward components referring to the positional relation of each of the coloured points to the respective black point "H" in Fig. 7. For each of the three arrangements A$\mathrm{C}$ in Fig. 7, the same computational procedure was applied 16 times, each time referring to another home site H (Supplement 12). Each of the points drawn in the graphs $\mathrm{Ab}-$ $\mathrm{Cb}(n=28$ per sample) refers, on the abscissa, to the original centre-related $\overline{\bar{c}}_{\mathrm{H}}$ value achieved with one of the $28 \mathrm{se}-$ lected VOC profiles and, on the ordinate, to the overall mean homeward component computed with the same VOC profile, but now with reference to any one of the 16 peripheral goal/home sites. As Fig. 7 shows, there are strong correlations $(r=0.82-0.92$; always $P<0.0001)$ between the original efficiency of such profiles iteratively achieved for homing to the centre and the efficiency now reached in orientation to peripheral goal sites. Thus, the weightings of atmospheric compounds being most useful for navigation to one geographic position are helpful for navigation to other positions as well.

It is, of course, a disadvantage that the geometry between release sites and a certain peripheral goal site can never be symmetric, so that goal-oriented compass directions as well as distances are not uniformly distributed (see examples $\mathrm{Aa}-\mathrm{Ca}$ in Fig. 7). Final symmetry is achieved, nevertheless, by use of 16 goal sites, each lying on one of the 16 radii along which the sampling sites were arranged. However, this symmetrisation cannot compensate for the fact that there is always, as seen from the goal site, a large majority of release sites on the side towards the centre, much 
fewer perpendicular to this axis and not one on the radius away from the centre. Thus, the goalward directions are not neutrally different from the directions used to develop the weighting profiles. It is possible, however, to distinguish between sites closer to and farther away from the axis running through the centre, respectively (green and red points in Fig. 7, Aa-Ca). Actually, achieved homeward components tend to be higher along rather than approximated perpendicular to that axis, but profits gained from centre-related optimisation are obvious also in the latter case (Fig. 7, $\mathrm{Ab}-\mathrm{Cb}$, red symbols). In accordance with the findings shown above, also navigation to peripheral sites functions better over long rather than over shorter distances ( $\mathrm{Cb}$ vs. $\mathrm{Bb}$ vs. $\mathrm{Ab}$ ).

\section{Discussion}

Experiments with real pigeons and other birds led to the conclusion that they are able to orient their courses approximately towards home from various distant sites all-around in unfamiliar areas by usage of atmospheric trace gases, homesite winds and a compass (see Introduction). At first sight, this conclusion appears unrealistic, as one would not expect that the chemical atmosphere contains the necessary spatial information. Now, however, realising that it is possible to create virtual birds that achieve just those navigational performances by making use of real trace gases and real winds, why should we consider it impossible for living birds to achieve a similar output by sensing a similar input? Mechanisms operating inside the black box between input and output may be very different in nature as compared with the model. Decisive at the present state is the apparent fact that the atmosphere does contain relevant positional information. If one mechanism is able to extract it in a suitable way, another mechanism may be able to do so as well. The algorithms used here do not imply the assumption that pigeons discriminate single compounds and are aware of ratios among them. Also we, if comparing flavours of different perfumes or wines, know nothing about the many involved chemical compounds and the ratios among them. The mechanism behind our conscious perception, however, must in some way evaluate these ratios. It translates different proportional compositions of a given set of compounds into differently sensed odour qualities. Pigeons might operate with similar perceptions or they might simply feel "I should fly this or that angle to the sun" without being aware of a certain smell at all.

The model calculations conducted on the basis of iteratively improved weighting and selection of most suitable VOCs demonstrate that the ratio-gradient hypothesis is not unrealistic; using the available atmospheric data, it works. Even more detailed predictions are met, namely expected kinds of dependence on distance from home and on concurrent winds on the days and at the sites of virtual release. The results are quite comparable to results obtained with living pigeons in nature. They even agree with the more specific ob- servation that homeward orientation of real pigeons housed at the central site of the investigated area tends to improve with increasing distance as well (Wallraff, 2005a, Fig. 3.5) and that such distance effects have been found also in other areas (Schmidt-Koenig, 1966, 1970).

Despite this general success, we should be aware of a number of restrictions and remaining open questions. (1) Unlike evolution, the iterative algorithm searching for optimised weightings of VOCs was focussed on only one geographic position as goal to be accessed. Naturally developed weightings should be more generally applicable to any goal in continent-wide dimensions. As a first approximation it could be shown, at least, that weightings optimised for orientation towards one goal are helpful for orientation towards other goals, not very distant, as well (Fig. 7). (2) The role of olfaction in the model is exclusively focussed on navigation. In reality, however, birds use odours in other contexts as well. Varying sensitivity to different substances alone may not be identical with the varying weightings produced by the model. Odours being relevant for other purposes, but irrelevant for true navigation, as far as they are present in the free atmosphere at all, should be sorted out or neutralised in some way. Further, the system is flexible enough to integrate arbitrary artificial odours which most likely are not naturally involved (Papi et al., 1974; Ioalè et al., 1990; see Fig. 7.25 in Wallraff, 2005a). Apparently, the set of navigationally exploitable compounds is not exclusively determined on a genetic basis. An artificial odour administered at home strongly correlated with a particular direction of artificial wind in an unnaturally high concentration, if again given upon displacement to a distant site, may outweigh and even mask all natural VOCs so that the pigeons have little chance to respond to any other olfactory input and thus follow this one dominant message. (3) It should be emphasised once more (see also W\&A) that certainly most of the 46 included VOCs are anthropogenic pollutants which were not available during evolution. Anyhow, more than half of the VOCs selected by the model as navigationally useful were larger molecules with at least eight carbon atoms (Fig. 3) whose concentration in the atmosphere was very low (Supplement 1: Table S1). Particularly this group of VOCs might contain also naturally emitted substances (e.g. monoterpenes) whose chemical identity has not been determined.

The model tackles the problems from the birds' point of view; it knows nothing about any regularity in spatial distributions of airborne compounds. For the birds, during evolution, it was sufficient to recognise that specific similarities of olfactory input perceived somewhere afield with corresponding input associated at home with winds from a certain direction indicates that their current position is in roughly that direction from their home. The birds may have optimised the system by adaptive sensitivity to particularly suitable chemosignals and neglect of others. The extension to hundreds of kilometres could not have been possible, however, if the chemical atmosphere would not have contained 
spatially ordered structures in these dimensions. Hardly anyone of us would have expected that such far-reaching and sufficiently stable regularities do exist in the unstable atmosphere. However, following the birds' advice rather than our intuitive assessment, we did actually find far-reaching ratio gradients of airborne trace gases (W\&A). The now most urgent problem to be solved is not a problem of biology, but of atmospheric chemistry and meteorology. How is it possible that proportional relationships among some particular VOCs vary gradually not only with varying winds, but even over such long distances over manifold landscapes and topographical features in the two-dimensional space (i.e. lowermost air layer) as well? Professional atmospheric chemistry has never asked this question (e.g. Williams, 2004), as there was no reason to focus any interest on spatial regularities of ratios among arbitrary VOCs which need not interact in any way and need not have anything else in common but just their concurrent presence in the air. It should be noted that lacking gradients in concentrations of single VOCs need not indicate a lack of ratio gradients among two or more VOCs (see Wallraff, 2001, Fig. 11, $C_{\mathrm{W}-\mathrm{E}}$ vs. $D_{\mathrm{W}-\mathrm{E}}$ ).

It was not the aim of this study to retrace the particular spatial and wind-related interrelationships among the navigationally most suitable VOCs. An analysis in this respect might perhaps deliver some insight into the intraatmospheric background making the results of this study possible. An initial approach has already been achieved earlier (Figs. 7 and 8 in W\&A): there are reasons to assume that the two-dimensional ratio pattern within the boundary layer is relatively stable in itself, but is displaced by winds and movements of air masses as a whole.

Over the years, studies on pigeon homing have revealed a number of peculiarities which may shed some light on the nature of the chemical-environmental background of avian goal-oriented navigation. In the following brief overview I refer to figures and related text in Wallraff, 2005a, where more detailed information and references can be found. (1) Regional variability. Results like those shown above could probably not be obtained in Tuscany/Italy. Over shorter distances $(<50 \mathrm{~km})$, pigeons are more clearly homeward oriented there than in Germany (Fig. 3.20), but over longer distances $(>100 \mathrm{~km})$ they often fail to orient homeward at all, if they were disabled to smell environmental air during the outward journey (Fig. 7.16). The first finding should probably be seen in the context of climatic conditions (higher evaporation rates from vegetation in the south?), the second in the context of geomorphology (Italy: opposing coasts only $200 \mathrm{~km}$ distant, mountainous land in between; Germany $200 \mathrm{~km}$ around Würzburg: no high mountains, no coast). High mountains (the Alps) between home site and release site can lead to systematic errors in olfactory navigation (Figs. 7.15, 7.35). Experiments with analogous olfactory deprivation were conducted around seven home sites in four continents; anosmic pigeons were always disoriented, intact controls showed different levels of homeward orientation (Fig. 7.4). (2) Local variability. In a site-specific manner, initial bearings of pigeons usually deviate more or less from a direct course towards home (similar to VPs here in Fig. 2). However, these so-called release-site biases reflect not exclusively responses to olfactory input (Wallraff, 1996; p. 48ff. in 2005a). (3) Temporal variability. In Germany, homing performances of pigeons showed a clear annual cycle roughly following the curve of air temperature (Figs. 3.16, 3.17), in autumn and winter even correlated with short-term fluctuations of temperature. Day-to-day variations and even hour-to-hour variations of initial orientation occur also intra-seasonal, at some sites more than at others (Figs. 3.3, 3.16, 3.19). (4) Air from different environments. Breathing air in little ventilated places among vegetation, e.g. in forests, resulted in reduced levels of homeward orientation compared with breathing air in an open landscape (Fig. 7.10). (5) Time course of signal processing. Pigeons must smell release-site air over a time span of more than a few minutes (apparently averaging olfactory input) in order to achieve full performance of homeward orientation (Fig. 7.9). (6) Altitudes of navigational cues above ground. Necessary information is available within a few metres above ground level. For effective evaluation of winds at home, pigeons can sit in an aviary not more than $4 \mathrm{~m}$ high. While waiting at a release site before departing homeward oriented under nasal anaesthesia, the birds can sit in a container at ground level or less than $2 \mathrm{~m}$ above it, obviously sensing there useful chemosignals. Altitudes of homing flights are usually below $100 \mathrm{~m}$. (7) Current winds during homing. Real pigeons (like here the VPs) do not deduce navigational information from concurrent winds at the release sites. Artificial winds during waiting there did not influence departure directions (unpublished negative results). An indirect coinciding conclusion can be drawn from clock-shift experiments (p. 527 in Wallraff, 1989).

These findings, being in good accordance with a navigation system based on airborne chemosignals, give some hints on its functionality. The topic "regional variability" animates to further speculations. If a relatively, but by no means completely flat land with a patchy topography, such as southern and central Germany, enables farther-reaching atmospheric ratio gradients to exist than the narrow mountainous Italy, would not the much more homogeneous oceans be candidates for even more extended and less disturbed regularities (cf. W\&A and p.177ff. in Wallraff, 2005a). Recently it has been shown that displaced shearwaters (procellariiform seabirds with particularly well-developed olfactory organs) returned to their home island quite fast and straight over $800 \mathrm{~km}$ of oceanic surface, but only if their sense of smell was unimpaired (Gagliardo et al., 2013).

There are lots of unsolved problems remaining. Which concrete trace gases do birds use for navigation? Can either biology or atmospheric chemistry recommend particular candidates? Are the involved compounds the same everywhere on earth? Over land and sea? Genetically fixed (species-specific?) or learned or a combination of both? At 
least some genetic basis should be expected if weighting of specific compounds, as implied by the model, is really important. Also, the mode of processing of olfactory signals in the context of navigation need not fully conform to our everyday experience with the sense of smell. For instance, the system should be permanently responsive without adaptation, but it is not a necessary precondition that a bird consciously smells the decisive olfactory input and hence that it responds to a particular "odour" in the sense in which we use this word.

\section{Outlook}

We are still far away from understanding goal-oriented longdistance navigation of pigeons and other birds. At least, however, it seems evident on which concrete questions we should focus our efforts of future research. We have a coherent collection of diverse empirical biological and environmental findings and we have a working hypothesis joining both kinds of findings together. Now, in addition, we have a strong support of this hypothesis letting its seemingly impossible part appear possible: the necessary spatial information can be extracted from the dynamic chemical atmosphere.

With this conclusion, the challenge is transferred from behavioural biology to air chemistry. Is it in any way possible to construct an atmospheric model which not only includes apparently existing ratio gradients covering distances of $400 \mathrm{~km}$ and more, but also explains their genesis and relative stability? If it is true that winds displace a fairly stable ratio pattern of some particular VOCs as a whole (see above), does this mean that the pattern must have a rather regional origin and does not depend on long-range transport of the decisive VOCs along more or less curved trajectories? Are there any data known, beyond those reported by $\mathrm{A} \& \mathrm{~W}$ and here, which might in some way touch the spatial information problem, although they were originally collected in another context? Is it possible to understand the spatial regularities in the relative abundance of the particular VOCs that were found to be "navigationally useful" and are chemically identified (labelled by +++ and ++ in Table S1)? Is it possible, at least hypothetically, to replace numbers of "useful" non-identified VOCs by plausible chemical compound names together with their sources, lifetimes, etc. and possible distributions? In short: can atmospheric chemistry, based on present knowledge, explain how it is possible that the observed ratio gradients do exist? If not, are any lines of future research conceivable that might lead to the origin of the observed ratio gradients?

The conclusion that birds navigate by exploiting olfactory input from the atmosphere has always suffered from a lack of immediate plausibility. Realising that there are spatial atmospheric structures which in fact can be exploited for home-finding makes the conclusion plausible for birds and biologists. However, the lack of immediate plausibility is not yet resolved, but merely dislocated to another discipline: how is it possible that a patchy irregular landscape is covered by an air layer implying fairly regular gradual changes of proportional relations among a number of trace gases which constitute a remarkably stable wide-ranging two-dimensional pattern?

The service charges for this open access publication have been covered by the Max Planck Society.

Edited by: J. Kesselmeier

\section{Supplementary material related to this article is available online at http://www.biogeosciences.net/10/ 6929/2013/bg-10-6929-2013-supplement.pdf.}

\section{References}

Able, K. P.: The debate over olfactory navigation by homing pigeons, J. Exp. Biol., 199, 121-124, 1996.

Able, K. P.: The concepts and terminology of bird navigation, J. Avian Biol., 32, 174-183, 2000.

Batschelet, E.: Circular statistics in biology. Academic Press, London, 1981.

Becker, J. and van Raden, H.: Meteorologische Gesichtspunkte zur olfaktorischen Navigationshypothese, J. Ornithol., 127, 1-8, 1986.

Boström, J. E., Åkesson, S., and Alerstam, T.: Where on earth can animals use a geomagnetic bi-coordinate map for navigation?, Ecography, 35, 1039-1047, 2012.

Gagliardo, A.: Forty years of olfactory navigation in birds, J. Exp. Biol., 216, 2165-2171, 2013.

Gagliardo, A., Bried, J., Lambardi, P., Luschi, P., Wikelski, M., and Bonadonna, F.: Oceanic navigation in Cory's shearwaters: evidence for a crucial role of olfactory cues for homing after displacement, J. Exp. Biol., 216, 2798-2805, 2013.

Ioalè, P., Nozzolini, M., and Papi, F.: Homing pigeons do extract directional information from olfactory stimuli, Behav. Ecol. Sociobiol., 26, 301-305, 1990.

Kramer, G.: Experiments on bird orientation, Ibis, 94, 265-285, 1952.

Merkel, F. W. and Wiltschko, W.: Magnetismus und Richtungsfinden zugunruhiger Rotkehlchen (Erithacus rubecula), Vogelwarte, 23, 71-77, 1965.

Papi, F.: Pigeon navigation: solved problems and open questions, Monit. Zool. Ital. (N. S.), 20, 471-517, 1986.

Papi, F.: Pigeons use olfactory cues to navigate, Ethol. Ecol. Evol., 1, 219-231, 1989.

Papi, F.: Olfactory navigation, in: Orientation in birds, edited by: Berthold, P., Birkhäuser, Basel, 52-85, 1991.

Papi, F., Fiore, L., Fiaschi, V., and Benvenuti, S.: The influence of olfactory nerve section on the homing capacity of carrier pigeons, Monit. Zool. Ital. (N.S.), 5, 265-267, 1971.

Papi, F., Ioalè, P., Fiaschi, V., Benvenuti, S., and Baldaccini, N. E.: Olfactory navigation of pigeons: the effect of treatment with odorous air currents, J. Comp. Physiol., 94, 187-193, 1974. 
Schmidt-Koenig, K.: Über die Entfernung als Parameter bei der Anfangsorientierung der Brieftaube, Z. vergl. Physiol., 52, 33-55, 1966.

Schmidt-Koenig, K.: Entfernung und Heimkehrverhalten der Brieftaube, Z. vergl. Physiol., 68, 39-48, 1970.

Waldvogel, J. A.: Olfactory navigation in homing pigeons: are the current models atmospherically realistic?, Auk, 104, 369-379, 1987.

Waldvogel, J. A.: Olfactory orientation in birds, Curr. Ornithol., 6, 269-321, 1989.

Wallraff, H. G.: Simulated navigation based on assumed gradients of atmospheric trace gases (models on pigeon homing, part 2), J. Theor. Biol., 138, 11-528, 1989.

Wallraff, H. G.: Navigation by homing pigeons, Ethol. Ecol. Evol., 2, 81-115, 1990.

Wallraff, H. G.: Seven theses on pigeon homing deduced from empirical findings, J. Exp. Biol., 199, 105-111, 1996.

Wallraff, H. G.: Simulated navigation based on observed gradients of atmospheric trace gases (models on pigeon homing, part 3), J. Theor. Biol., 205, 133-145, 2000.
Wallraff, H. G.: Navigation by homing pigeons: updated perspective, Ethol. Ecol. Evol., 13, 1-48, 2001.

Wallraff, H. G.: Avian olfactory navigation: its empirical foundation and conceptual state, Anim. Behav., 67, 189-204, 2004.

Wallraff, H. G.: Avian navigation: pigeon homing as a paradigm, Springer, Berlin, 2005a.

Wallraff, H. G.: Beyond familiar landmarks and integrated routes: goal-oriented navigation by birds, Connect. Sci, 17, 91-106, 2005b.

Wallraff, H. G.: Pigeon homing as a model case of goal-oriented navigation, in: Encyclopedia of animal behavior, vol 2, edited by: Breed, M. D. and Moore, J., Academic Press, Oxford, 713-722, 2010.

Wallraff, H. G. and Andreae, M. O.: Spatial gradients in ratios of atmospheric trace gases: a study stimulated by experiments on bird navigation, Tellus, 52, 1138-1157, 2000.

Williams, J.: Organic trace gases in the atmosphere: an overview, Environ. Chem., 1, 125-136, 2004. 